Sharif University of Technology
Scientia Iranica
Transactions E: Industrial Engineering
http://scientiairanica.sharif.edu
IRAN ICA

\title{
A novel correlation coefficient of intuitionistic fuzzy sets based on the connection number of set pair analysis and its application
}

\author{
H. Garg* and K. Kumar \\ School of Mathematics, Thapar Institute of Engineering \& Technology (Deemed University), Patiala 147004, Punjab, India.
}

Received 1 November 2016; received in revised form 11 February 2017; accepted 17 July 2017

\author{
KEYWORDS \\ Set pair analysis; \\ Connection number; \\ Intuitionistic fuzzy \\ set; \\ Pattern recognition; \\ Medical diagnosis; \\ Decision-making.
}

\begin{abstract}
Set Pair Analysis (SPA) is an updated theory of dealing with uncertainty, which overlaps with other theories of uncertainty such as probability, vague, fuzzy, and Intuitionistic Fuzzy Set (IFS). Considering the fact that the correlation coefficient plays an important role during a decision-making process, after revealing the weakness of the existing correlation coefficients between the IFSs, this study proposes a novel correlation coefficient and weighted correlation coefficients formulation to measure the relative strength of different IFSs. To do so, firstly, corresponding to each intuitionistic fuzzy number, the connection number of the SPA theory is formulated in the form of the identity, discrepancy, and contrary degrees; moreover, measurements of the novel correlation coefficient are defined. Pairs of identity, discrepancy, and contrary of the connection number are taken as a vector representation during the formulation. Lastly, a decision-making approach based on the proposed measures is presented, illustrated by two numerical examples in pattern recognition and medical diagnosis.
\end{abstract}

(C) 2018 Sharif University of Technology. All rights reserved.

\section{Introduction}

Success of human beings in life depends on the ability of decision-making (DM) and recognition of precise situations, demonstrating that DM plays an important role in human life. However, due to the increasing complexity of the system, it is difficult for decisionmakers to make a perfect decision, since most of preferences and values during the DM process are imbued with uncertainty. To handle it, Zadeh [1] introduced the concept of Fuzzy Set (FS) theory to deal with uncertain or ambiguous data by assigning a membership value corresponding to each element

\footnotetext{
*. Corresponding author.

E-mail address: harishg58iitr@gmail.com (H. Garg)
}

doi: $10.24200 /$ sci.2017.4454 whose range is between 0 and 1 . Following their successful study, researchers have become preoccupied, in their extensions and out of them, with Intuitionistic Fuzzy Set (IFS) and Interval-Valued Intuitionistic Fuzzy Set (IVIFS) theories, as proposed by Atanassov [2] and Atanassov and Gargov [3], respectively. They involved the membership and non-membership degrees simultaneously, and many scholars have widely used them. Research on this subject can be classified roughly into four main topics: aggregation operators, measures, decision-making methods, and preference relation. For instance, $\mathrm{Xu}$ and Yager [4] presented a geometric aggregation operator, while $\mathrm{Xu}$ [5] presented a weighted averaging operator for aggregating different Intuitionistic Fuzzy Numbers (IFNs). Garg [6] presented a generalized intuitionistic fuzzy interactive geometric aggregation operator using Einstein t-norm and t-conorm operations. $\mathrm{Xu}$ and Chen [7], $\mathrm{Xu}$ [8] 
developed some arithmetic and geometric aggregation operators, namely interval-valued intuitionistic fuzzy weighted averaging and geometric operators, respectively, for aggregating the interval-valued intuitionistic fuzzy information. Garg et al. [9] presented an entropybased approach to solving the decision-making problem under fuzzy environment. Garg [10], further, developed a new generalized, improved score function to rank the IVIFSs. Later on, Garg [11,12] extended the theory of the IFS to the Pythagorean fuzzy set, which is less than one, to relax the condition of the sum of their membership function to square sum of its membership functions. He presented generalized geometric as well as averaging aggregation operators. Apart from that, various researchers pay more attention to the decisionmaking process to aggregate different alternatives using different aggregation operators [13-23] and their corresponding references.

On the other hand, a correlation plays an important role in statistics and engineering sciences. Through correlation analysis, the joint relationship of two variables can be examined with the aid of a measure of interdependency of the two variables. Chiang and Lin [24] discussed the correlation of fuzzy data based on conventional statistics and derived a formula for the correlation coefficient of fuzzy sets. Liu and Kao [25] studied the correlation coefficient of fuzzy numbers using a mathematical programming approach based on the classical definition of correlation coefficients. Later, Hong [26] studied the correlation coefficient of fuzzy numbers by applying $T_{w}$-based (the weakest t-norm) algebraic operations. Wang and $\mathrm{Li}$ [27] introduced the correlation of intervalvalued fuzzy numbers. Furthermore, Gerstenkorn and Manko [28] introduced the correlation coefficient of IFSs, whereas Bustince and Burillo [29] discussed the concepts of correlation and correlation coefficient of Interval-Valued Intuitionistic Fuzzy Sets (IVIFSs). Hong [30] generalized the concepts of correlation and correlation coefficient of IVIFSs to a general probability space and extended the results of Bustince and Burillo [29]. Xu et al. [31] defined a correlation coefficient of IFSs from the set-theoretic viewpoint. $\mathrm{Xu}$ [32] provided a survey on correlation analysis of IFSs and proposed a new method for deriving the correlation coefficients of IFSs, enjoying some advantages over the existing methods. Garg [33] defined the concepts of correlation and correlation coefficients of Pythagorean fuzzy sets. Garg [34], further, presented a novel accuracy function under the interval-valued Pythagorean fuzzy set to solve the decision-making problems.

Based on these studies, it has been observed that their proposed correlation coefficients have several drawbacks. For instance, measures of the existing correlation coefficients are independent of the degree of the membership or non-membership when one of the membership and non-membership degrees is zero in IFSs. In addition, the effect of a change of the degree of the membership function on their index values remains unaffected. Therefore, it gives an inconsistent model which is unable to rank different IFSs on the respective scales. Although the above studies have been widely used by researchers, their credibility is not guaranteed. In order to handle the uncertainties in a more precise way, Zhao [35] introduced the Set Pair Analysis (SPA) for handling the uncertainties specific to the data in which certainty and uncertainty are studied as one system. SPA is a modified uncertainty theory in which both certainties and uncertainties are treated as an integrated certain-uncertain system. In SPA, the main component is the Connection Number (CN) which represents three aspects of identity, discrepancy, and contrary degrees to describe the certainty and uncertainty of an element. The main principal of SPA is to analyze the feature of set pair and construct a connection degree for them. The concept of the arithmetic operations between the connection numbers was defined in Liu et al. [36]. Wang and Gong [37] proposed a decision-making method based on the set-pair analysis to solve the decision-making problems with ascertained criteria weight and the criteria value being an interval random variable. $\mathrm{Hu}$ and Yang [38] proposed a dynamic stochastic multi-attribute decisionmaking approach based on the cumulative prospect theory and SPA. Xie et al. [39] presented a CN under an interval-valued fuzzy set by taking the positive and negative ideal schemes. Fu and Zhou [40] proposed a triangular fuzzy MCDM method based on SPA. Kumar and Garg [41] presented a Technique for Order of Preference by Similarity to Ideal Solution (TOPSIS) method under the IVIFS environment based on the connection number of the SPA. Apart from these, some researchers [42-50] have solved the fuzzy decisionmaking problem under SPA.

Thus, based on the above observations, it has been observed that SPA is one of the most successful theories of handling the uncertainties and certainties in the system. Motivated by this, the present paper develops the correlation coefficient measures based on the connection number of the SPA to solve the multi-criteria group decision-making problems where preferences related to different alternatives are taken in the form of the IFNs. In order to achieve it, firstly, the shortcoming of the existing correlation coefficient measures under the IFSs environment has been highlighted; then, connection numbers corresponding to IFNs have been proposed to overcome these shortcomings. Based on these connection numbers, a new informational energy and the covariance between the two CNs have been defined; hence, the correlation coefficient and a weighted correlation coefficient have been proposed. Then, accordingly, the problem of MCDM from the 
fields of pattern recognition and medical diagnosis has been solved to validate the effectiveness and applicability of the proposed decision method.

The rest of the paper is organized as follows. Section 2 describes the basic concepts of the IFSs and their corresponding existing correlation coefficients. Section 3 presents correlation coefficients and weighted correlation coefficients based on the connection number of the SPA. Section 4 highlights the shortcomings of the existing correlation coefficients. Section 5 describes the decision-making method based on the proposed correlation coefficients under the SPA theory. Section 6 demonstrates the proposed correlation coefficients with numerical examples related to pattern recognition and medical diagnoses. Finally, Section 7 presents a concrete conclusion.

\section{Preliminaries}

In this section, some basic concepts about the IFSs are defined over non-empty universal set $X$.

\subsection{Intuitionistic Fuzzy Set (IFS)}

An IFS $A$ is defined as a set of ordered pairs over a universal set $X$ given by [2]:

$$
A=\left\{\left\langle x, u_{A}(x), v_{A}(x)\right\rangle \mid x \in X\right\}
$$

where $u_{A}: X \rightarrow[0,1]$ and $v_{A}: X \rightarrow[0,1]$ represent the degrees of membership and non-membership of $x$ to $A$ such that for any $x \in X, u_{A}(x)+v_{A}(x) \leq 1$. Moreover, the intuitionistic index of $x$ to $A$ is defined as $\pi_{A}(x)=$ $1-u_{A}(x)-v_{A}(x)$, Usually, the pair $\left\langle u_{A}(x), v_{A}(x)\right\rangle$ is called an Intuitionistic Fuzzy Number (shorted by IFN) and is often simplified as $\langle u, v\rangle$ where $u \in[0,1], v \in$ $[0,1], u+v \leq 1$.

\subsection{Correlation coefficient based on IFSs}

For any two IFSs $A=\left\{\left\langle x_{t}, u_{A}\left(x_{t}\right), v_{A}\left(x_{t}\right)\right\rangle \mid x_{t} \in X\right\}$ and $B=\left\{\left\langle x_{t}, u_{B}\left(x_{t}\right), v_{B}\left(x_{t}\right)\right\rangle \mid x_{t} \in X\right\}$ in the universe of discourse $X$, the informational energies of these sets are denoted by $E_{I F S}(A)$ and $E_{I F S}(B)$, respectively, which are defined as follows [28]:

$$
\begin{aligned}
& E_{I F S}(A)=\sum_{t=1}^{n}\left[u_{A}^{2}\left(x_{t}\right)+v_{A}^{2}\left(x_{t}\right)\right] \\
& E_{I F S}(B)=\sum_{t=1}^{n}\left[u_{B}^{2}\left(x_{t}\right)+v_{B}^{2}\left(x_{t}\right)\right] .
\end{aligned}
$$

The correlation of IFSs $A$ and $B$ is defined as follows:

$$
C_{I F S}(A, B)=\sum_{t=1}^{n}\left(u_{A}\left(x_{t}\right) u_{B}\left(x_{t}\right)+v_{A}\left(x_{t}\right) v_{B}\left(x_{t}\right)\right) \text {. }
$$

Thus, accordingly, the correlation coefficients of $A$ and $B$ are defined as follows:

$$
\begin{aligned}
& K_{I F S_{1}}(A, B)=\frac{C_{I F S}(A, B)}{\sqrt{E_{I F S}(A) \cdot E_{I F S}(B)}} \\
& =\frac{\sum_{t=1}^{n}\left(u_{A}\left(x_{t}\right) u_{B}\left(x_{t}\right)+v_{A}\left(x_{t}\right) v_{B}\left(x_{t}\right)\right)}{\sqrt{\sum_{t=1}^{n}\left(u_{A}^{2}\left(x_{t}\right)+v_{A}^{2}\left(x_{t}\right)\right) \cdot \sum_{t=1}^{n}\left(u_{B}^{2}\left(x_{t}\right)+v_{B}^{2}\left(x_{t}\right)\right)}} .
\end{aligned}
$$

On the other hand, Xu et al. [31] suggested an alternative form of the correlation coefficients of IFSs $A$ and $B$ by Eqs. (2) and (3) as shown in Box I. The function $K_{I F S}$ satisfies the following properties:

(P1) $0 \leq K_{I F S}(A, B) \leq 1$;

(P2) $K_{I F S}(A, B)=K_{I F S}(B, A)$;

(P3) $K_{I F S}(A, B)=1$ if $A=B$.

Example 2.1. Let $A_{1}, A_{2}$, and $A_{3}$ be three IFSs defined in $X=\left\{x_{1}, x_{2}\right\}$ by: $A_{1}=\left\{\left(x_{1}, 0.10,0.15\right)\right.$, $\left.\left(x_{2}, 0.10,0.15\right)\right\}, \quad A_{2}=\left\{\left(x_{1}, 0.20,0.30\right), \quad\left(x_{2}, 0.20\right.\right.$, $0.30)\}$, and $A_{3}=\left\{\left(x_{1}, 0.30,0.45\right),\left(x_{2}, 0.30,0.45\right)\right\}$. Then, it is easy to check if $K_{I F S_{1}}\left(A_{1}, A_{2}\right)=$ $K_{I F S_{1}}\left(A_{2}, A_{3}\right)=K_{I F S_{1}}\left(A_{3}, A_{1}\right)$.

$$
K_{I F S_{2}}(A, B)=\frac{\sum_{t=1}^{n}\left(u_{A}\left(x_{t}\right) u_{B}\left(x_{t}\right)+v_{A}\left(x_{t}\right) v_{B}\left(x_{t}\right)\right)}{\max \left\{\left\{\sum_{t=1}^{n}\left(u_{A}^{2}\left(x_{t}\right)+v_{A}^{2}\left(x_{t}\right)\right)\right\}^{1 / 2},\left\{\sum_{t=1}^{n}\left(u_{B}^{2}\left(x_{t}\right)+v_{B}^{2}\left(x_{t}\right)\right)\right\}^{1 / 2}\right\}},
$$

and:

$$
K_{I F S_{3}}(A, B)=\frac{\sum_{t=1}^{n}\left(u_{A}\left(x_{t}\right) u_{B}\left(x_{t}\right)+v_{A}\left(x_{t}\right) v_{B}\left(x_{t}\right)+\pi_{A}\left(x_{t}\right) \pi_{B}\left(x_{t}\right)\right)}{\max \left\{\sum_{t=1}^{n}\left(u_{A}^{2}\left(x_{t}\right)+v_{A}^{2}\left(x_{t}\right)+\pi_{A}^{2}\left(x_{t}\right)\right), \sum_{t=1}^{n}\left(u_{B}^{2}\left(x_{t}\right)+v_{B}^{2}\left(x_{t}\right)+\pi_{B}^{2}\left(x_{t}\right)\right)\right\}} .
$$


Example 2.2. Consider two IFNs $A=\langle 0.4,0\rangle$ and $B=\langle 0,0.5\rangle$. Then, the correlation coefficient between them, by using Eq. (1), is $K_{I F S_{1}}(A, B)=$ $\frac{(0.4)(0)+(0.5)(0)}{\sqrt{0.4^{2}+0^{2}} \sqrt{0.5^{2}+0^{2}}}=0$. On the other hand, if we replace IFN $A$ with $A_{1}=\langle 0.6,0\rangle$, then the correlation coefficient between $A_{1}$ and $B$ is $K_{I F S_{1}}\left(A_{1}, B\right)=$

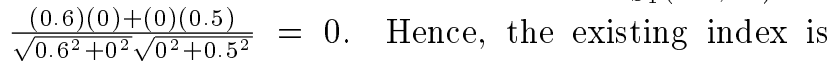
independent of the change of the membership degree in IFS.

Example 2.3. Let two IFNs $A=\langle 0.4,0.3\rangle$ and $B=$ $\langle 0,0.5\rangle$, then the correlation coefficient becomes:

$$
K_{I F S_{1}}(A, B)=\frac{(0.4)(0)+(0.3)(0.5)}{\sqrt{0.4^{2}+0.3^{2}} \sqrt{0^{2}+0.5^{2}}}=0.6 \text {. }
$$

On the other hand, if IFNs $A$ and $B$ are replaced with $A_{1}=\langle 0.1,0.4898\rangle$ and $B_{1}=\langle 0.45189,0.21398\rangle$, respectively, then the correlation coefficient becomes:

$$
\begin{aligned}
& K_{I F S_{1}}\left(A_{1}, B_{1}\right)= \\
& \quad \frac{(0.1)(0.45189)+(0.4898)(0.21398)}{\sqrt{0.1^{2}+0.4898^{2}} \sqrt{0.45189^{2}+0.21398^{2}}}=0.6 .
\end{aligned}
$$

Hence, it has been concluded that, by changing the membership and non-membership degrees of IFSs, the correlation coefficient remains the same; therefore, it is inconsistent and, hence, unable to rank the alternatives. Therefore, there is a need to enhance these measures.

\subsection{Set pair analysis}

Zhao [35] presented a novel concept of the Set Pair Analysis (SPA) to deal with both of the certainty and uncertainty degrees as one system based on the quantitative analysis. The main principal of SPA is to analyze the features of set pair and construct a connection degree for them. The set pair of two interrelated sets $A$ and $B$ denoted by $H(A, B)$ for a given problem $W$ and Connection Number $(\mathrm{CN})$, represented by $\mu(H, W)$, is characterized by the the three components, namely "identity", "discrepancy", and "contrary", and are defined as follows:

$$
\mu(H, W)=(S / N)+(F / N) i+(P / N) j,
$$

where $N$ is the total number of features, in which $S$ represents the "identity" features, $P$ is the "contrary" features and $F=N-S-P$ is the "discrepancy" features of sets $A$ and $B$. Herein, we define the ratio $S / N=a$ (identity degree), $F / N=b$ (discrepancy degree), and $P / N=c$ (contrary degree). Then, Eq. (4) becomes:

$$
\mu(H, W)=a+b i+c j .
$$

Herein, $i$ is coefficient of "discrepancy degree" such that $i \in[-1,1], j$ is the coefficient of "contrary degree", and $j=-1$. It is clearly seen that $0 \leq a, b, c \leq 1$ and $a+b+c=1$.

\section{The proposed correlation coefficient based on connection numbers}

Let $A=\langle u, v\rangle$ be an IFS where $u$ and $v$ represent the degrees of membership and nonmembership values, respectively, of $A$ such that $u, v \in[0,1]$ and $u+v \leq 1$. Then, connection number $\mu$ corresponding to set $A$ is defined as follows:

$$
\mu(H, A)=a+b i+c j,
$$

where $a=u(1-v)$ is the "identity", $b=1-u(1-v)-$ $v(1-u)$ is the "discrepancy", and $c=v(1-u)$ is the "contrary" degrees.

Let $A=\left\{\left\langle x_{t}, u_{A}\left(x_{t}\right), v_{A}\left(x_{t}\right)\right\rangle \mid x_{t} \in X\right\}$ be an IFS in the universe of discourse $X$, and its corresponding connection number is defined as $\mu_{A}=\left\{a\left(x_{t}\right)+b\left(x_{t}\right) i+\right.$ $\left.c\left(x_{t}\right) j \mid x_{t} \in X\right\}$ where $a\left(x_{t}\right), b\left(x_{t}\right), c\left(x_{t}\right) \in[0,1]$ and $a\left(x_{t}\right)+b\left(x_{t}\right)+c\left(x_{t}\right)=1$; for every $x_{t} \in X$, we define:

$$
E(A)=\sum_{t=1}^{n}\left(a^{2}\left(x_{t}\right)+b^{2}\left(x_{t}\right)+c^{2}\left(x_{t}\right)\right),
$$

which is called the informational energy of set $A$.

Assume that two sets:

$$
A=\left\{\left(x_{t}, a_{1}\left(x_{t}\right), b_{1}\left(x_{t}\right), c_{1}\left(x_{t}\right)\right) \mid x_{t} \in X\right\},
$$

and:

$$
B=\left\{\left(x_{t}, a_{2}\left(x_{t}\right), b_{2}\left(x_{t}\right), c_{2}\left(x_{t}\right)\right) \mid x_{t} \in X\right\},
$$

are defined over the universe of discourse $X$ where $a_{1}\left(x_{t}\right), a_{2}\left(x_{t}\right), b_{1}\left(x_{t}\right), b_{2}\left(x_{t}\right), c_{1}\left(x_{t}\right), c_{2}\left(x_{t}\right) \in[0,1]$ for every $x_{t} \in X$. Then, the following so-called correlation of CNs $A$ and $B$ is defined as follows:

$$
\begin{aligned}
C(A, B)= & \sum_{t=1}^{n}\left[a_{1}\left(x_{t}\right) \cdot a_{2}\left(x_{t}\right)+b_{1}\left(x_{t}\right) \cdot b_{2}\left(x_{t}\right)\right. \\
& \left.+c_{1}\left(x_{t}\right) \cdot c_{2}\left(x_{t}\right)\right] .
\end{aligned}
$$

From the above, it is obvious that the correlation of CNs satisfies the following properties:

(P1) $C(A, A)=E(A)$;

(P2) $C(A, B)=C(B, A)$.

Definition 3.1. Let $A$ and $B$ be the sets of CNs corresponding to the IFSs on a universe of discourse $X=\left\{x_{1}, x_{2}, \ldots, x_{n}\right\}$ denoted by $A=$ $\left\{\left(x_{t}, a_{1}\left(x_{t}\right), b_{1}\left(x_{t}\right), c_{1}\left(x_{t}\right)\right) \mid x_{t} \in X\right\}$ and $B=$ $\left\{\left(x_{t}, a_{2}\left(x_{t}\right), b_{2}\left(x_{t}\right), c_{2}\left(x_{t}\right)\right) \mid x_{t} \in X\right\}$, respectively. Then, the correlation coefficient between $A$ and $B$ is given by Eq. (9) as shown in Box II.

Theorem 3.1. For any two sets of CNs $A$ and $B$ defined over the universe of discourse $X=$ $\left\{x_{1}, x_{2}, \ldots, x_{n}\right\}$, correlation coefficient $K_{1}$ satisfies the following properties. 


$$
K_{1}(A, B)=\frac{C(A, B)}{\sqrt{E(A) \cdot E(B)}}=\frac{\sum_{t=1}^{n}\left[a_{1}\left(x_{t}\right) \cdot a_{2}\left(x_{t}\right)+b_{1}\left(x_{t}\right) \cdot b_{2}\left(x_{t}\right)+c_{1}\left(x_{t}\right) \cdot c_{2}\left(x_{t}\right)\right]}{\sqrt{\sum_{t=1}^{n}\left(a_{1}^{2}\left(x_{t}\right)+b_{1}^{2}\left(x_{t}\right)+c_{1}^{2}\left(x_{t}\right)\right)} \sqrt{\sum_{t=1}^{n}\left(a_{2}^{2}\left(x_{t}\right)+b_{2}^{2}\left(x_{t}\right)+c_{2}^{2}\left(x_{t}\right)\right)}} .
$$

Box II

(P1) $K_{1}(A, B)=K_{1}(B, A)$;

(P2) $0 \leq K_{1}(A, B) \leq 1$;

(P3) $A=B \Leftrightarrow K_{1}(A, B)=1$;

(P4) If $A \subseteq B \subseteq C$, for a connection number $C$ then $K_{1}(A, C) \leq K_{1}(A, B)$ and $K_{1}(A, C) \leq$ $K_{1}(B, C)$.

\section{Proof.}

(P1) It is straightforward.

(P2) The inequality in $K_{1}(A, B) \geq 0$ is evident; thus, as shown in Box III we prove $K_{1}(A, B) \leq 1$. By Cauchy-Schwarz inequality, we have:

$$
\begin{gathered}
\left(x_{1} y_{1}+x_{2} y_{2}+\ldots+x_{n} y_{n}\right)^{2} \\
\leq\left(x_{1}^{2}+y_{1}^{2}+\ldots+x_{n}^{2}\right) \\
\cdot\left(y_{1}^{2}+y_{2}^{2}+\ldots+y_{n}^{2}\right)
\end{gathered}
$$

where $\left(x_{1}+x_{2}+\ldots+x_{n}\right) \in R^{n}$ and $\left(y_{1}+y_{2}+\ldots+\right.$ $\left.y_{n}\right) \in R^{n}$. Then we obtain the relation shown in Box IV.

Let the following notations be:

$$
\sum_{t=1}^{n} a_{1}^{2}\left(x_{t}\right)=\xi_{1}, \quad \sum_{t=1}^{n} b_{1}^{2}\left(x_{t}\right)=\rho_{1}
$$

$$
\begin{array}{ll}
\sum_{t=1}^{n} c_{1}^{2}\left(x_{t}\right)=\eta_{1}, & \sum_{t=1}^{n} a_{2}^{2}\left(x_{t}\right)=\xi_{2}, \\
\sum_{t=1}^{n} b_{2}^{2}\left(x_{t}\right)=\rho_{2}, & \sum_{t=1}^{n} c_{2}^{2}\left(x_{t}\right)=\eta_{2} .
\end{array}
$$

So the above inequality becomes:

$$
K_{1}(A, B) \leq \frac{\sqrt{\xi_{1} \xi_{2}}+\sqrt{\rho_{1} \rho_{2}}+\sqrt{\eta_{1} \eta_{2}}}{\sqrt{\xi_{1}+\rho_{1}+\eta_{1}} \cdot \sqrt{\xi_{2}+\rho_{2}+\eta_{2}}} .
$$

By squaring on both sides, we get:

$$
K_{1}^{2}(A, B) \leq \frac{\left(\sqrt{\xi_{1} \xi_{2}}+\sqrt{\rho_{1} \rho_{2}}+\sqrt{\eta_{1} \eta_{2}}\right)^{2}}{\left(\xi_{1}+\rho_{1}+\eta_{1}\right) \cdot\left(\xi_{2}+\rho_{2}+\eta_{2}\right)} .
$$

By subtracting 1 from both sides, we obtain the relation shown in Box V. Thus, we have $0 \leq$ $K_{1}(A, B) \leq 1$.

(P3) Sufficient condition is obviously true. Now, necessary condition is proven.

So, let equality sign hold in the proof of (P2) if we have $a_{1}\left(x_{t}\right)=h a_{2}\left(x_{t}\right), b_{1}\left(x_{t}\right)=$ $h b_{2}\left(x_{t}\right)$ and $c_{1}\left(x_{t}\right)=h c_{2}\left(x_{t}\right)$ for some positive real number $h$. However, according to the condition of $\mathrm{CN}$, we have $a_{1}\left(x_{t}\right)+b_{1}\left(x_{t}\right)+c_{1}\left(x_{t}\right)=$

$$
K_{1}(A, B)=\frac{\sum_{t=1}^{n}\left(a_{1}\left(x_{t}\right) \cdot a_{2}\left(x_{t}\right)+b_{1}\left(x_{t}\right) \cdot b_{2}\left(x_{t}\right)+c_{1}\left(x_{t}\right) \cdot c_{2}\left(x_{t}\right)\right)}{\sqrt{\sum_{t=1}^{n}\left(a_{1}^{2}\left(x_{t}\right)+b_{1}^{2}\left(x_{t}\right)+c_{1}^{2}\left(x_{t}\right)\right) \cdot \sum_{t=1}^{n}\left(a_{2}^{2}\left(x_{t}\right)+b_{2}^{2}\left(x_{t}\right)+c_{2}^{2}\left(x_{t}\right)\right)}}
$$

\section{Box III}

$$
K_{1}(A, B) \leq \frac{\left(\sum_{t=1}^{n} a_{1}^{2}\left(x_{t}\right) \sum_{t=1}^{n} a_{2}^{2}\left(x_{t}\right)\right)^{1 / 2}+\left(\sum_{t=1}^{n} b_{1}^{2}\left(x_{t}\right) \sum_{t=1}^{n} b_{2}^{2}\left(x_{t}\right)\right)^{1 / 2}+\left(\sum_{t=1}^{n} c_{1}^{2}\left(x_{t}\right) \sum_{t=1}^{n} c_{2}^{2}\left(x_{t}\right)\right)^{1 / 2}}{\sqrt{\left(\sum_{t=1}^{n} a_{1}^{2}\left(x_{t}\right)+\sum_{t=1}^{n} b_{1}^{2}\left(x_{t}\right)+\sum_{t=1}^{n} c_{1}^{2}\left(x_{t}\right)\right)} \cdot \sqrt{\left(\sum_{t=1}^{n} a_{2}^{2}\left(x_{t}\right)+\sum_{t=1}^{n} b_{2}^{2}\left(x_{t}\right)+\sum_{t=1}^{n} c_{2}^{2}\left(x_{t}\right)\right)}}
$$




$$
K_{1}^{2}(A, B)-1 \leq \frac{-\left(\left(\sqrt{\xi_{1} \rho_{2}}-\sqrt{\rho_{1} \xi_{2}}\right)^{2}+\left(\sqrt{\rho_{1} \eta_{2}}-\sqrt{\eta_{1} \rho_{2}}\right)^{2}+\left(\sqrt{\xi_{1} \eta_{2}}-\sqrt{\eta_{1} \xi_{2}}\right)^{2}\right)}{\left(\xi_{1}+\rho_{1}+\eta_{1}\right) \cdot\left(\xi_{2}+\rho_{2}+\eta_{2}\right)} \leq 0 .
$$

\section{Box $\mathrm{V}$}

$1=a_{2}\left(x_{t}\right)+b_{2}\left(x_{t}\right)+c_{2}\left(x_{t}\right)$ which implies that $h=1$. Hence, $A=B$.

(P4) $A \subseteq B \subseteq C$ which implies that $a_{1}\left(x_{t}\right) \leq$ $a_{2}\left(x_{t}\right) \leq a_{3}\left(x_{t}\right), b_{1}\left(x_{t}\right) \geq b_{2}\left(x_{t}\right) \geq b_{3}\left(x_{t}\right)$, and $c_{1}\left(x_{t}\right) \geq c_{2}\left(x_{t}\right) \geq c_{3}\left(x_{t}\right)$. Geometrically, if $A \subseteq B \subseteq C$, then the angle between $A$ and $C$ should be larger than the angles between $A$ and $B$ and between $B$ and $C$ for any element $x_{t}$ and is a decreasing function within the interval $[0, \pi / 2]$. Thus, relations $K_{1}(A, C) \leq K_{1}(A, B)$ and $K_{1}(A, C) \leq K_{1}(B, C)$ can be obtained by Eq. (9)

Example 3.1. Let $A$ and $B$ be any two sets of CNs defined in $X=\left\{x_{1}, x_{2}, x_{3}\right\}$ such that $A=\left\{\left(x_{1}, 0.25\right.\right.$, $\left.0.35,0.40),\left(x_{2}, 0.30,0.45,0.25\right),\left(x_{3}, 0.60,0.20,0.20\right)\right\}$, $B=\left\{\left(x_{1}, 0.75,0.15,0.10\right),\left(x_{2}, 0.55,0.35,0.10\right)\right.$, and $\left.\left(x_{3}, 0.40,0.20,0.40\right)\right\}$. Then, using Eq. (7), the informational energy of $A$ is written as follows:

$$
\begin{aligned}
E(A)= & \sum_{t=1}^{n}\left(a_{1}^{2}\left(x_{t}\right)+b_{1}^{2}\left(x_{t}\right)+c_{1}^{2}\left(x_{t}\right)\right) \\
= & \left(0.25^{2}+0.35^{2}+0.40^{2}\right) \\
& +\left(0.30^{2}+0.45^{2}+0.25^{2}\right) \\
& +\left(0.60^{2}+0.20^{2}+0.20^{2}\right)=1.1400 .
\end{aligned}
$$

The informational energy of $B$ is given as $E(B)=$ 1.3900. Now, by using Eq. (8), the correlation between sets $A$ and $B$ is written as follows:

$$
\begin{aligned}
C(A, B)= & \sum_{t=1}^{n}\left[a_{1}\left(x_{t}\right) \cdot a_{2}\left(x_{t}\right)+b_{1}\left(x_{t}\right) \cdot b_{2}\left(x_{t}\right)\right. \\
& \left.+c_{1}\left(x_{t}\right) \cdot c_{2}\left(x_{t}\right)\right]=(0.25 \times 0.75 \\
& +0.35 \times 0.15+0.40 \times 0.10) \\
& +(0.30 \times 0.55+0.45 \times 0.35 \\
& +0.25 \times 0.10)+(0.60 \times 0.40 \\
& +0.20 \times 0.20+0.20 \times 0.40)=0.9875 .
\end{aligned}
$$

Hence, the correlation coefficient between the sets of CNs $A$ and $B$ is given by:

$$
\begin{aligned}
K_{1}(A, B) & =\frac{C(A, B)}{\sqrt{E(A) \cdot E(B)}}=\frac{0.9875}{1.1400 \times 1.3900} \\
& =0.7845 .
\end{aligned}
$$

Furthermore, in order to stress the importance of the proposed correlation coefficient with respect to the existing ones, the correlation measure has been computed for the above taken examples, where the existing measures have failed to rank the alternatives, as below.

Example 3.2. If the proposed measure is applied to the above considered Example 2.1, then we get $K_{1}\left(A_{1}, A_{2}\right)=0.9759, \quad K_{1}\left(A_{2}, A_{3}\right)=0.9839$ and $K_{1}\left(A_{3}, A_{1}\right)=0.9215$. Thus, the proposed measure classifies the IFSs and, hence, is able to identify the best one.

Example 3.3. If the proposed correlation coefficient measure is applied to Example 2.2, i.e., by taking two IFNs $A=\langle 0.4,0\rangle$ and $B=\langle 0,0.5\rangle$ then $K_{1}(A, B)=$ 0.5883 . On the other hand, if IFN $A$ is replaced with $A_{1}=\langle 0.6,0\rangle$, then the correlation coefficient between $A_{1}$ and $B$ is $K_{1}\left(A_{1}, B\right)=0.3922$. Hence, the proposed measure simultaneously considers the effect of the change of the membership degree on IFS during the analysis.

Example 3.4. If the proposed measures are applied to the data as considered in Example 2.3, then we get $K_{1}(A, B)=0.8026$, and if the replaced IFNs are used, $A_{1}=\langle 0.1,0.4898\rangle$ and $B_{1}=\langle 0.45189,0.21398\rangle$, then we get $K_{1}\left(A_{1}, B_{1}\right)=0.7865$. Therefore, the proposed measure has sufficiently considered the impact of the change in the degrees of membership and non-membership on the correlation coefficient measure and, hence, has overcome the shortcomings of the existing measures.

Definition 3.2. Let $A$ and $B$ be the set of two CNs. Then, the correlation coefficient is defined by Eq. (10) as shown in Box VI.

Theorem 3.2. Correlation coefficient, $K_{2}(A, B)$, between the two sets of $\mathrm{CNs} A$ and $B$, satisfies the following properties:

(P1) $K_{2}(A, B)=K_{2}(B, A)$; 


$$
K_{2}(A, B)=\frac{C(A, B)}{\max \{E(A), E(B)\}}=\frac{\sum_{t=1}^{n}\left[a_{1}\left(x_{t}\right) \cdot a_{2}\left(x_{t}\right)+b_{1}\left(x_{t}\right) \cdot b_{2}\left(x_{t}\right)+c_{1}\left(x_{t}\right) \cdot c_{2}\left(x_{t}\right)\right]}{\max \left\{\sum_{t=1}^{n}\left(a_{1}^{2}\left(x_{t}\right)+b_{1}^{2}\left(x_{t}\right)+c_{1}^{2}\left(x_{t}\right)\right), \sum_{t=1}^{n}\left(a_{2}^{2}\left(x_{t}\right)+b_{2}^{2}\left(x_{t}\right)+c_{2}^{2}\left(x_{t}\right)\right)\right\}} .
$$

Box VI

$$
K_{3}(A, B)=\frac{C_{\omega}(A, B)}{\sqrt{E_{\omega}(A) \cdot E_{\omega}(B)}}=\frac{\sum_{t=1}^{n} \omega_{t}\left(a_{1}\left(x_{t}\right) \cdot a_{2}\left(x_{t}\right)+b_{1}\left(x_{t}\right) \cdot b_{2}\left(x_{t}\right)+c_{1}\left(x_{t}\right) \cdot c_{2}\left(x_{t}\right)\right)}{\sqrt{\sum_{t=1}^{n} \omega_{t}\left(a_{1}^{2}\left(x_{t}\right)+b_{1}^{2}\left(x_{t}\right)+c_{1}^{2}\left(x_{t}\right)\right) \cdot \sum_{t=1}^{n} \omega_{t}\left(a_{2}^{2}\left(x_{t}\right)+b_{2}^{2}\left(x_{t}\right)+c_{2}^{2}\left(x_{t}\right)\right)}},
$$

and:

$$
K_{4}(A, B)=\frac{C_{\omega}(A, B)}{\max \left\{E_{\omega}(A), E_{\omega}(B)\right\}}=\frac{\sum_{t=1}^{n} \omega_{t}\left(a_{1}\left(x_{t}\right) \cdot a_{2}\left(x_{t}\right)+b_{1}\left(x_{t}\right) \cdot b_{2}\left(x_{t}\right)+c_{1}\left(x_{t}\right) \cdot c_{2}\left(x_{t}\right)\right)}{\max \left\{\sum_{t=1}^{n} \omega_{t}\left(a_{1}^{2}\left(x_{t}\right)+b_{1}^{2}\left(x_{t}\right)+c_{1}^{2}\left(x_{t}\right)\right), \sum_{t=1}^{n} \omega_{t}\left(a_{2}^{2}\left(x_{t}\right)+b_{2}^{2}\left(x_{t}\right)+c_{2}^{2}\left(x_{t}\right)\right)\right\}} .
$$

Box VII

(P2) $0 \leq K_{2}(A, B) \leq 1$

(P3) $A=B \Leftrightarrow K_{2}(A, B)=1$;

(P4) If $A \subseteq B \subseteq C$ for a connection number $C$ then $\bar{K}_{2}(A, \bar{C}) \leq K_{2}(A, B)$ and $K_{2}(A, C) \leq$ $K_{2}(B, C)$.

Proof. Properties (P1), (P3), and (P4) are straight forward; thus, we omit them here. Also, $K_{2}(A, B) \geq$ 0 is evident. Now, from Theorem 3.1, we have $(C(A, B))^{2} \leq E(A) \cdot E(B)$. Therefore, $C(A, B) \leq$ $\max \{E(A), E(B)\} ;$ thus, $K_{2}(A, B) \leq 1 . \square$

However, in many practical situations, different sets may have taken different weights; thus, weight $\omega_{t}$ of element $x_{t} \in X(t=1,2, \ldots, n)$ should be taken into account such that $\omega_{t}>0, \sum_{t=1}^{n} \omega_{t}=1$. In the following, a weighted correlation coefficient is developed between CNs in which by Eqs. (11) and (12) as shown in Box VII, the above formulated $K_{1}(A, B)$ and $K_{2}(A, B)$ are extended to the weighted correlation coefficient.

It can be easily deduced that if $\omega=(1 / n$, $1 / n, \ldots, 1 / n)^{T}$, then Eqs. (11) and (12) reduce to the correlation coefficient defined in Eqs. (9) and (10), respectively. It is easy to see that the weighted correlation coefficients $K_{3}(A, B)$ and $K_{4}(A, B)$ between the sets of CNs satisfy the properties of $0 \leq K_{3}(A, B) \leq 1$ and $0 \leq K_{4}(A, B) \leq 1$.

Theorem 3.3. Let $\omega=\left(\omega_{1}, \omega_{2}, \ldots, \omega_{n}\right)^{T}$ be the weight vector of $x_{t}(t=1,2, \ldots, n)$, such that $\omega_{t}>$ 0 and $\sum_{t} \omega_{t}=1$. Then, the weighted correlation coefficient, $K_{k}(A, B)(k=3,4)$, between the sets of CNs $A$ and $B$ defined by Eqs. (11) and (12), satisfies the following properties:

(P1) $K_{k}(A, B)=K_{k}(B, A)$;

(P2) $0 \leq K_{k}(A, B) \leq 1$;

(P3) $A=B \Leftrightarrow K_{k}(A, B)=1$;

(P4) If $A \subseteq B \subseteq C$ for a connection number $C$, then $K_{k}(A, C) \leq K_{k}(A, B)$ and $K_{k}(A, C) \leq$ $K_{k}(B, C)$.

Proof. Proof follows the above theorem; hence, it is omitted here.

\section{Drawbacks of the existing correlation coefficients}

In this section, some limitations inherent in the existing methods of correlation coefficients for Atanassov IFSs are shown. Advantages of the proposed correlation coefficients have been discussed. To show the limitations of the existing correlation coefficients, a few examples are presented in this section. For convenience, assume that the weights of elements, $x_{t} \in X$, are all equal.

4.1. Correlation coefficient of Zeng and Li [51] Assume that there are three patterns defined in the form of IFS with the degrees of membership and non- 


$$
Z L(A, B)=\frac{\sum_{t=1}^{n}\left(u_{A}\left(x_{t}\right) u_{B}\left(x_{t}\right)+v_{A}\left(x_{t}\right) v_{B}\left(x_{t}\right)+\pi_{A}\left(x_{t}\right) \pi_{B}\left(x_{t}\right)\right)}{\sqrt{\sum_{t=1}^{n}\left(u_{A}^{2}\left(x_{t}\right)+v_{A}^{2}\left(x_{t}\right)+\pi_{A}^{2}\left(x_{t}\right)\right) \cdot \sum_{t=1}^{n}\left(u_{B}^{2}\left(x_{t}\right)+v_{B}^{2}\left(x_{t}\right)+\pi_{B}^{2}\left(x_{t}\right)\right)}} .
$$

\section{Box VIII}

membership $\left\langle u\left(x_{t}\right), v\left(x_{t}\right)\right\rangle$ in $X=\left\{x_{1}, x_{2}, x_{3}\right\}$ defined as follows:

$$
\begin{aligned}
& A_{1}=\left\{\left\langle x_{1}, 0.4,0.5\right\rangle,\left\langle x_{2}, 0.7,0.1\right\rangle,\left\langle x_{3}, 0.3,0.3\right\rangle\right\}, \\
& A_{2}=\left\{\left\langle x_{1}, 0.5,0.4\right\rangle,\left\langle x_{2}, 0.7,0.2\right\rangle,\left\langle x_{3}, 0.4,0.3\right\rangle\right\}, \\
& A_{3}=\left\{\left\langle x_{1}, 0.4,0.5\right\rangle,\left\langle x_{2}, 0.7,0.1\right\rangle,\left\langle x_{3}, 0.4,0.3\right\rangle\right\} .
\end{aligned}
$$

Assume that a sample of $B=\left\{\left\langle x_{1}, 0.1,0.1\right\rangle,\left\langle x_{2}, 1.0\right.\right.$, $\left.0.0\rangle,\left\langle x_{3}, 0.0,1.0\right\rangle\right\}$ is given.

Zeng and $\mathrm{Li}$ [51] defined the correlation measure between IFSs $A$ and $B$ by Eq. (13) as shown in Box VIII. Hence, accordingly, the suitable classifier for sample $B$ from $A_{1}, A_{2}$ and $A_{3}$ patterns can be identified using Eq. (13). The results corresponding to each pattern are obtained as follows:

$$
Z L\left(A_{1}, B\right)=Z L\left(A_{2}, B\right)=Z L\left(A_{3}, B\right)=0.6262 .
$$

On the other hand, to identify which pattern belongs to sample B by using Eq. (9), we convert the intuitionistic fuzzy values of the given patterns and sample into the CNs according to Eq. (6) as follows:

$$
\begin{aligned}
A_{1}= & \left\{\left\langle x_{1}, 0.20,0.50,0.30\right\rangle,\left\langle x_{2}, 0.63,0.34,0.03\right\rangle,\right. \\
& \left.\left\langle x_{3}, 0.21,0.58,0.21\right\rangle\right\}, \\
A_{2}= & \left\{\left\langle x_{1}, 0.30,0.50,0.20\right\rangle,\left\langle x_{2}, 0.56,0.38,0.06\right\rangle,\right. \\
& \left.\left\langle x_{3}, 0.28,0.54,0.18\right\rangle\right\}, \\
A_{3}= & \left\{\left\langle x_{1}, 0.20,0.50,0.30\right\rangle,\left\langle x_{2}, 0.63,0.34,0.03\right\rangle,\right. \\
& \left.\left\langle x_{3}, 0.28,0.54,0.18\right\rangle\right\}, \\
B= & \left\{\left\langle x_{1}, 0.09,0.82,0.09\right\rangle,\left\langle x_{2}, 1.00,0.00,0.00\right\rangle,\right. \\
& \left.\left\langle x_{3}, 0.00,0.00,1.00\right\rangle\right\} .
\end{aligned}
$$

Then, based on these CNs, Eq. (9) is utilized, and the measure values are obtained as follows:

$$
\begin{aligned}
& K_{1}\left(A_{1}, B\right)=0.6879, \\
& K_{1}\left(A_{2}, B\right)=0.6534, \\
& K_{1}\left(A_{3}, B\right)=0.6777 .
\end{aligned}
$$

Thus, based on these results, correlation coefficient of Zeng and $\mathrm{Li}$ [51] cannot classify this sample. On the other hand, the SPA theory is able to accommodate the required information about the sample and, hence, find if sample $B$ belongs to pattern $A_{1}$ according to the recognition principle.

\subsection{Correlation coefficient of Szmidt and Kacprzyk [52]}

Szmidt and Kacprzyk [52] proposed a correlation coefficient for two Atanassov's IFSs, $A$ and $B$, to express not only a relative strength but also a positive or negative relationship between $A$ and $B$. Suppose that there exists a random sample $x_{1}, x_{2}, \ldots, x_{n} \in X$. Then, correlation coefficient $r(A, B)$ is given by:

$$
r(A, B)=\frac{r_{1}(A, B)+r_{2}(A, B)+r_{3}(A, B)}{3},
$$

where:

$$
\begin{aligned}
& r_{1}(A, B)=\frac{\sum_{t=1}^{n}\left(u_{A}\left(x_{t}\right)-\overline{u_{A}}\right)\left(u_{B}\left(x_{t}\right)-\overline{u_{B}}\right)}{\sqrt{\sum_{t=1}^{n}\left(u_{A}\left(x_{t}\right)-\overline{u_{A}}\right)^{2} \cdot \sum_{t=1}^{n}\left(u_{B}\left(x_{t}\right)-\overline{u_{B}}\right)^{2}}}, \\
& r_{2}(A, B)=\frac{\sum_{t=1}^{n}\left(v_{A}\left(x_{t}\right)-\overline{v_{A}}\right)\left(v_{B}\left(x_{t}\right)-\overline{v_{B}}\right)}{\sqrt{\sum_{t=1}^{n}\left(v_{A}\left(x_{t}\right)-\overline{v_{A}}\right)^{2} \cdot \sum_{t=1}^{n}\left(v_{B}\left(x_{t}\right)-\overline{v_{B}}\right)^{2}}}, \\
& r_{3}(A, B)=\frac{\sum_{t=1}^{n}\left(\pi_{A}\left(x_{t}\right)-\overline{\pi_{A}}\right)\left(\pi_{B}\left(x_{t}\right)-\overline{\pi_{B}}\right)}{\sqrt{\sum_{t=1}^{n}\left(\pi_{A}\left(x_{t}\right)-\overline{\pi_{A}}\right)^{2} \cdot \sum_{t=1}^{n}\left(\pi_{B}\left(x_{t}\right)-\overline{\pi_{B}}\right)^{2}}},
\end{aligned}
$$

where:

$$
\begin{aligned}
\overline{u_{A}} & =\frac{1}{n} \sum_{t=1}^{n} u_{A}\left(x_{t}\right), & \overline{u_{B}} & =\frac{1}{n} \sum_{t=1}^{n} u_{B}\left(x_{t}\right), \\
\overline{v_{A}} & =\frac{1}{n} \sum_{t=1}^{n} v_{A}\left(x_{t}\right), & \overline{v_{B}} & =\frac{1}{n} \sum_{t=1}^{n} v_{B}\left(x_{t}\right), \\
\overline{\pi_{A}} & =\frac{1}{n} \sum_{t=1}^{n} \pi_{A}\left(x_{t}\right), & \overline{\pi_{B}} & =\frac{1}{n} \sum_{t=1}^{n} \pi_{B}\left(x_{t}\right) .
\end{aligned}
$$

Based on the above formulated correlation coefficient, 
as shown, it is not able to find a good correlation between the intuitionistic fuzzy sets. For instance, consider $A$ and $B$ as two Atanassov's IFSs in $X=$ $\left\{x_{1}, x_{2}, x_{3}\right\}$ defined as:

$$
\begin{aligned}
& A=\left\{\left\langle x_{1}, 0.1,0.2\right\rangle,\left\langle x_{2}, 0.2,0.1\right\rangle,\left\langle x_{3}, 0.29,0.0\right\rangle\right\} \\
& B=\left\{\left\langle x_{1}, 0.1,0.3\right\rangle,\left\langle x_{2}, 0.2,0.2\right\rangle,\left\langle x_{3}, 0.29,0.1\right\rangle\right\} .
\end{aligned}
$$

Then, by applying Eq. (14), we get $r(A, B)=1$, which means that these two IFSs are perfectly correlated, which is not true as seen from the structure of sets $A$ and $B$. On the other hand, if our proposed method is applied to find the correlation coefficient between $A$ and $B$ using Eqs. (9) and (10), then we get $K_{1}(A, B)=$ 0.9922 and $K_{2}(A, B)=0.9298$, respectively. These results show that $A$ and $B$ are not perfectly correlated.

\subsection{Correlation coefficient of Xu et al. [31]}

$\mathrm{Xu}$ et al. [31] defined the correlation coefficient in the environment of intuitionistic fuzzy sets by considering the degrees of membership and non-membership as well as the degree of hesitation between the two intuitionistic sets $A$ and $B$ given in Box IX.

Consider the following three patterns $A_{1}, A_{2}$ and $A_{3}$ represented in the form of intuitionistic fuzzy sets:

$$
\begin{aligned}
& A_{1}=\left\{\left\langle x_{1}, 0.4,0.5\right\rangle,\left\langle x_{2}, 0.7,0.1\right\rangle,\left\langle x_{3}, 0.3,0.3\right\rangle\right\}, \\
& A_{2}=\left\{\left\langle x_{1}, 0.5,0.4\right\rangle,\left\langle x_{2}, 0.7,0.2\right\rangle,\left\langle x_{3}, 0.4,0.3\right\rangle\right\}, \\
& A_{3}=\left\{\left\langle x_{1}, 0.4,0.5\right\rangle,\left\langle x_{2}, 0.7,0.1\right\rangle,\left\langle x_{3}, 0.4,0.3\right\rangle\right\}
\end{aligned}
$$

Assume that a sample $B=\left\{\left\langle x_{1}, 0.1,0.1\right\rangle,\left\langle x_{2}, 1,0\right\rangle\right.$, $\left.\left\langle x_{3}, 0,1\right\rangle\right\}$ is given. Then, by using the correlation coefficient of $\mathrm{Xu}$ et al. [31] defined in Eq. (15), the following results are obtained:

$$
X u_{1}\left(A_{1}, B\right)=X u_{1}\left(A_{2}, B\right)=X u_{1}\left(A_{3}, B\right)=0.4398 .
$$

Thus, based on their results, it has been concluded that their correlation coefficient cannot be used to classify sample $B$ with three patterns $A_{1}, A_{2}$, and $A_{3}$.

To remove this problem, the intuitionistic fuzzy values of the given patterns and sample are converted into the CNs according to Eq. (6) as follows:

$$
\begin{aligned}
& A_{1}=\left\{\left\langle x_{1}, 0.20,0.50,0.30\right\rangle,\left\langle x_{2}, 0.63,0.34,0.03\right\rangle,\right. \\
&\left.\left\langle x_{3}, 0.21,0.58,0.21\right\rangle\right\}, \\
& A_{2}=\left\{\left\langle x_{1}, 0.30,0.50,0.20\right\rangle,\left\langle x_{2}, 0.56,0.38,0.06\right\rangle,\right. \\
&\left.\left\langle x_{3}, 0.28,0.54,0.18\right\rangle\right\}, \\
& A_{3}=\left\{\left\langle x_{1}, 0.20,0.50,0.30\right\rangle,\left\langle x_{2}, 0.63,0.34,0.03\right\rangle,\right. \\
&\left.\left\langle x_{3}, 0.28,0.54,0.18\right\rangle\right\}, \\
& B=\left\{\left\langle x_{1}, 0.09,0.82,0.09\right\rangle,\left\langle x_{3}, 1.00,0.00,0.00\right\rangle,\right. \\
&\left.\left\langle x_{3}, 0.00,0.00,1.00\right\rangle\right\} .
\end{aligned}
$$

Eq. (10) is utilized to obtain the correlation coefficient as follows:

$$
\begin{aligned}
& K_{2}\left(A_{1}, B\right)=0.4817, \\
& K_{2}\left(A_{2}, B\right)=0.4445, \\
& K_{2}\left(A_{3}, B\right)=0.4705 .
\end{aligned}
$$

Thus, it has been concluded that sample $B$ can be classified with pattern $A_{1}$.

\section{Decision-making approach based on the proposed correlation coefficients}

This section parents a method for the decision-making problems by means of the proposed correlation coefficient or weighted correlation coefficient between two or more IFSs. In this respect, assume that there are $m$ alternatives, denoted by $A_{1}, A_{2}, \ldots, A_{m}$, which are evaluated under the set of $n$ criteria, denoted by $C_{1}, C_{2}, \ldots, C_{n}$, and their preferences are given in the form of IFNs $\alpha_{k t}=\left\langle u_{k t}, v_{k t}\right\rangle ; k=1,2, \ldots, m ; t=$ $1,2, \ldots, n$ where $u_{k t}$ and $v_{k t}$, respectively, represent the degree of how much $A_{k}$ can satisfy and dissatisfy criteria $C_{t}$ such that $u_{k t}+v_{k t} \leq 1$. Thus, the rating values corresponding to each alternative are represented in the form of IFNs over the universal set $X$ as follows:

$$
\begin{aligned}
& A_{k}=\left\{\left\langle x_{t}, u_{k 1}\left(x_{t}\right), v_{k 1}\left(x_{t}\right)\right\rangle \mid x_{t} \in U\right\} \\
& k=1,2, \ldots, m ; \quad t=1,2, \ldots, n
\end{aligned}
$$

$$
X u_{1}(A, B)=\frac{\sum_{t=1}^{n}\left(u_{A}\left(x_{t}\right) u_{B}\left(x_{t}\right)+v_{A}\left(x_{t}\right) v_{B}\left(x_{t}\right)+\pi_{A}\left(x_{t}\right) \pi_{B}\left(x_{t}\right)\right)}{\max \left\{\sum_{t=1}^{n}\left(u_{A}^{2}\left(x_{t}\right)+v_{A}^{2}\left(x_{t}\right)+\pi_{A}^{2}\left(x_{t}\right)\right), \sum_{t=1}^{n}\left(u_{B}^{2}\left(x_{t}\right)+v_{B}^{2}\left(x_{t}\right)+\pi_{B}^{2}\left(x_{t}\right)\right)\right\}} .
$$


Let $\omega_{t}(t=1,2, \ldots, n)$ be the weight of criterion $C_{t}$ such that $\omega_{t}>0$ and $\sum_{t=1}^{n} \omega_{t}=1$. In the decisionmaking process, the concept of an ideal point has been used to access the best alternative. Although the ideal alternative does not exist in the real world, it does provide a useful theoretical construct to evaluate the alternatives. Therefore, each ideal alternative $A^{*}$ is proposed as IFN $\alpha_{t}^{*}=\langle 1,0\rangle$ for $t=1,2, \ldots, n$. Then, various steps involved in the proposed approach to finding the best alternative(s) are summarized as follows:

Step 1: Collect all the information corresponding to each alternative in terms of IFNs. Hence, an overall intuitionistic fuzzy decision matrix $D$ is expressed as follows:

$D=\left(\begin{array}{cccc}\left\langle u_{11}, v_{11}\right\rangle & \left\langle u_{12}, v_{12}\right\rangle & \ldots & \left\langle u_{1 n}, v_{1 n}\right\rangle \\ \left\langle u_{21}, v_{21}\right\rangle & \left\langle u_{22}, u_{22}\right\rangle & \ldots & \left\langle u_{2 n}, u_{2 n}\right\rangle \\ \vdots & \vdots & \ddots & \vdots \\ \left\langle u_{m 1}, v_{m 1}\right\rangle & \left\langle u_{n 2}, v_{n 2}\right\rangle & \ldots & \left\langle u_{m n}, v_{m n}\right\rangle\end{array}\right)$

Step 2: Convert this intuitionistic fuzzy normalized decision matrix into its equivalent connection number as $\mu_{k t}=a_{k t}+b_{k t} i+c_{k t} j$ where $a_{k t}=u_{k t}\left(1-v_{k t}\right)$, $b_{k t}=1-u_{k t}\left(1-v_{k t}\right)-v_{k t}\left(1-u_{k t}\right)$ and $c_{k t}=v_{k t}(1-$ $\left.u_{k t}\right)$.

Step 3: Compute $K_{1}\left(A_{k}, A^{*}\right)$ or $K_{2}\left(A_{k}, A^{*}\right)$ or $K_{3}\left(A_{k}, A^{*}\right)$ or $K_{4}\left(A_{k}, A^{*}\right)$ measure between alternatives $A_{k}(k=1,2, \ldots, m)$ and the ideal alternative $A^{*}$ using Eqs. (9), (10), (11) and (12), respectively, where $A^{*}$ is the ideal alternative whose connection number is given by $\mu_{A^{*}}=1+0 i+0 j$.

Step 4: Rank all of the alternatives with respect to the value of $K_{1}\left(A_{k}, A^{*}\right)$ or $K_{2}\left(A_{k}, A^{*}\right)$ or $K_{3}\left(A_{k}, A^{*}\right)$ or $K_{4}\left(A_{k}, A^{*}\right)(k=1,2, \ldots, m)$.

Step 5: Choose the best alternative with respect to the maximum value of $K_{1}\left(A_{k}, A^{*}\right)$ or $K_{2}\left(A_{k}, A^{*}\right)$ or $K_{3}\left(A_{k}, A^{*}\right)$ or $K_{4}\left(A_{k}, A^{*}\right)(k=1,2, \ldots, m)$.

\section{Illustrative Example}

In this section, an example related to the decisionmaking problem, from the field of pattern recognition and medical diagnosis, is taken to demonstrate the effectiveness of the proposed method.

Example 6.1: Pattern Recognition. Consider three known patterns $C_{1}, C_{2}$, and $C_{3}$ represented in the form of IFSs on $X=\left\{x_{1}, x_{2}, x_{3}\right\}$ as follows:

$$
\begin{aligned}
& C_{1}=\left\{\left\langle x_{1}, 1.0,0.0\right\rangle,\left\langle x_{2}, 0.8,0.0\right\rangle,\left\langle x_{3}, 0.7,0.1\right\rangle\right\}, \\
& C_{2}=\left\{\left\langle x_{1}, 0.8,0.1\right\rangle,\left\langle x_{2}, 1.0,0.0\right\rangle,\left\langle x_{3}, 0.9,0.0\right\rangle\right\}, \\
& C_{3}=\left\{\left\langle x_{1}, 0.6,0.2\right\rangle,\left\langle x_{2}, 0.8,0.0\right\rangle,\left\langle x_{3}, 1.0,0.0\right\rangle\right\} .
\end{aligned}
$$

Consider an unknown pattern $B$ which will be recognized, where:

$$
B=\left\{\left\langle x_{1}, 0.5,0.3\right\rangle,\left\langle x_{2}, 0.6,0.2\right\rangle,\left\langle x_{3}, 0.8,0.1\right\rangle\right\} .
$$

The target of this problem is to classify pattern $B$ in one of classes $C_{1}, C_{2}$ and $C_{3}$. To do so, firstly, the IFSs are converted to $\mathrm{CNs}$ of given patterns; then, sample and their corresponding numbers are summarized as below:

$$
\begin{aligned}
& C_{1}=\left\{\left\langle x_{1}, 1.0,0.0,0.0\right\rangle,\left\langle x_{2}, 0.8,0.20,0.0\right\rangle,\right. \\
&\left.\left\langle x_{3}, 0.63,0.34,0.03\right\rangle\right\}, \\
& C_{2}=\left\{\left\langle x_{1}, 0.72,0.26,0.02\right\rangle,\left\langle x_{2}, 1.0,0.0,0.0\right\rangle,\right. \\
&\left.\left\langle x_{3}, 0.9,0.1,0.0\right\rangle\right\} \\
& C_{3}=\left\{\left\langle x_{1}, 0.48,0.44,0.08\right\rangle,\left\langle x_{2}, 0.8,0.20,0.0\right\rangle,\right. \\
&\left.\left\langle x_{3}, 1.0,0.0,0.0\right\rangle\right\}, \\
& B=\left\{\left\langle x_{1}, 0.35,0.50,0.15\right\rangle,\left\langle x_{2}, 0.48,0.44,0.08\right\rangle,\right. \\
&\left.\left\langle x_{3}, 0.72,0.26,0.02\right\rangle\right\} .
\end{aligned}
$$

Now, the proposed correlation coefficient indexes, $K_{1}$ and $K_{2}$, have been computed from $B$ to $C_{k}(k=$ $1,2,3)$, and their results are given as follows:

$$
\begin{array}{ll}
K_{1}\left(C_{1}, B\right)=0.7755, & K_{1}\left(C_{2}, B\right)=0.8350, \\
K_{1}\left(C_{3}, B\right)=0.9223, & K_{2}\left(C_{1}, B\right)=0.6221, \\
K_{2}\left(C_{2}, B\right)=0.6395, & K_{2}\left(C_{3}, B\right)=0.7544 .
\end{array}
$$

Thus, from these two proposed correlation coefficient indexes, it can be concluded that pattern $B$ belongs to pattern $C_{3}$.

On the other hand, if it is assumed that weights of $x_{1}, x_{2}$ and $x_{3}$ are $0.5,0.3$, and 0.2 , respectively, then correlation coefficients $K_{3}$ and $K_{4}$ can be utilized for obtaining the most suitable pattern as follows:

$$
\begin{array}{ll}
K_{3}\left(C_{1}, B\right)=0.7104, & K_{3}\left(C_{2}, B\right)=0.8129, \\
K_{3}\left(C_{3}, B\right)=0.9262, & K_{4}\left(C_{1}, B\right)=0.5270, \\
K_{4}\left(C_{2}, B\right)=0.6224, & K_{4}\left(C_{3}, B\right)=0.7842 .
\end{array}
$$

Thus, ranking order of the three patterns is $C_{3}, C_{2}$, and $C_{1}$; hence, $C_{3}$ is the most desirable pattern classified with $B$.

In order to compare the performance of the proposed approach with those of some existing approaches under the IFS environment, a comparison analysis was conducted based on different approaches as given by the authors in $[31,51,53-57]$. The results are summarized as below: 
(a) If the correlation coefficient measures, as proposed by $\mathrm{Li}$ [51] given in Eq. (13), are applied to the considered problem, then their corresponding index values of pattern $C_{k}(k=1,2,3)$ are $Z L\left(C_{1}, B\right)=$ $0.8882, Z L\left(C_{2}, B\right)=0.9291$, and $Z L\left(C_{3}, B\right)=$ 0.9710 . Thus, ranking of the alternative is $C_{3} \succ$ $C_{2} \succ C_{1}$; hence, $C_{3}$ is the most desirable pattern classified with $B$;

(b) If the cosine similarity measure $C S M(\cdot)$, as proposed by Ye [53], is applied to the considered problem (for more details, see [53]), then its value corresponding to each pattern is $C S M\left(C_{1}, B\right)=0.9353, \operatorname{CSM}\left(C_{2}, B\right)=0.9519$, and $C S M\left(C_{3}, B\right)=0.9724$. Thus, ranking order is $C_{3} \succ C_{2} \succ C_{1}$; hence, the best alternative for classifying pattern $B$ is $C_{3}$;

(c) If similarity measure $S_{D C}(\cdot)$, as proposed by Dengfeng and Chuntian [54], is applied to the considered data then its index values for pattern $C_{k}(k=1,2,3)$ with respect to unknown pattern $B$ is computed as $S_{D C}\left(C_{1}, B\right)=0.74, S_{D C}\left(C_{2}, B\right)=$ 0.78 , and $S_{D C}\left(C_{3}, B\right)=0.84$, while by Liu [55] measure denoted by $T(\cdot)$ (for more details, see $[55]), T\left(C_{1}, B\right)=0.72, T\left(C_{2}, B\right)=0.74$, and $T\left(C_{3}, B\right)=0.84$ are obtained. Thus, $C_{3}$ is the most desirable pattern classified with $B$;

(d) If the correlation coefficient, as proposed by $\mathrm{Xu}$ et al. [31], given in Eq. (15), is applied to the considered problem, then the index values corresponding to these measures are $X u_{1}\left(C_{1}, B\right)=$ $0.7252, X u_{1}\left(C_{2}, B\right)=0.7177$, and $X u_{1}\left(C_{3}, B\right)=$ 0.8113 . Therefore, $C_{3}$ is the most desirable pattern classified with $B$;

(e) If distance measure $d(\cdot)$, as proposed by Ejegwa and Modom [56], is applied to the considered problem (for more details, see [56]), then the measure values corresponding to each pattern are obtained as follows: $d\left(C_{1}, B\right)=0.3200, d\left(C_{2}, B\right)=0.3200$, and $d\left(C_{3}, B\right)=0.2000$. Therefore, $C_{3}$ is the most desirable pattern classified with $B$;

(f) If similarity measure $S(\cdot)$, as proposed by Hung and Yang [57], is applied to the considered problem, then their respective measure values corresponding to each pattern are obtained, $S\left(C_{1}, B\right)=$ $0.4700, S\left(C_{2}, B\right)=0.4700$, and $S\left(C_{3}, B\right)=$ 0.5100 ; hence, the ranking of these pattern is $C_{3} \succ C_{2}=C_{1}$. Therefore, pattern $C_{3}$ is classified with pattern $B$.

Example 6.2: Medical diagnosis. Consider the dataset in [58] containing four patients: $P=\{A l, B o b$, Joe, Ted $\}$, five symptoms: $S=\left\{s_{1}\right.$ (Tempera- ture), $s_{2}$ (HeadAche), $s_{3}$ (Stomach Pain), $s_{4}$ (Cough), $s_{5}$ (Chest pain) $\}$, and five diseases: $Q=\left\{Q_{1}\right.$ (Viral fever), $Q_{2}$ (Malaria), $Q_{3}$ (Typhoid), $Q_{4}$ (Stomach Problem), $Q_{5}$ (Chest problem) $\}$. The relations between the patientsthe symptoms, denoted by $P S$, and the symptoms-the diseases, denoted by $S Q$, are represented in the form of the IFNs as shown in Box X. Then, the target of this problem is to classify patients $P=\left\{A l, B o b, J_{o e}, T e d\right\}$ with one of diagnoses $Q=\left\{Q_{1}, Q_{2}, Q_{3}, Q_{4}, Q_{5}\right\}$, respectively. In order to apply the proposed approach to the considered data, firstly, the information related to the patients with respect to the symptoms and the diagnoses with respect to the symptoms are converted into the connection numbers, and their corresponding results are summarized as shown in Box XI. Now, a developed correlation coefficient $K_{1}$ as given in Eq. (9) has been utilized for the above data; hence, the follow-

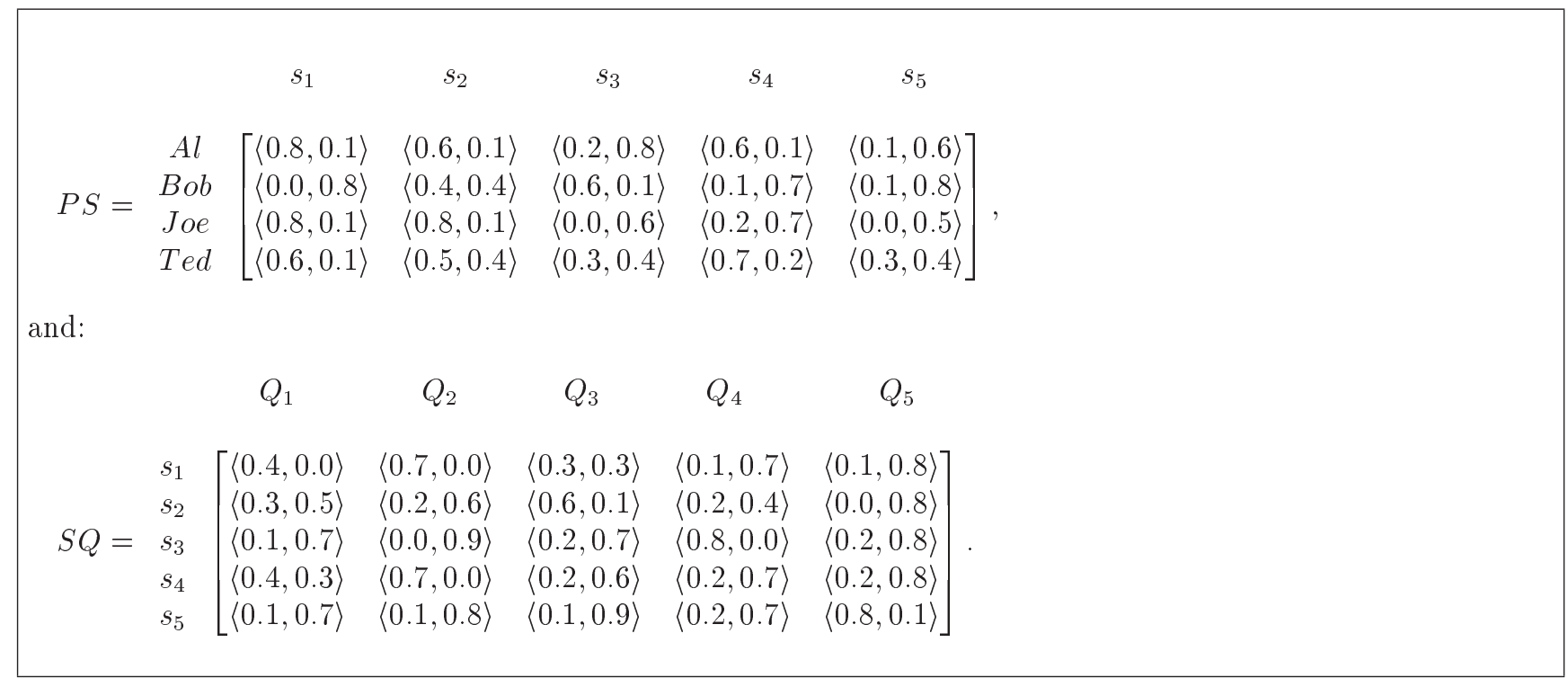




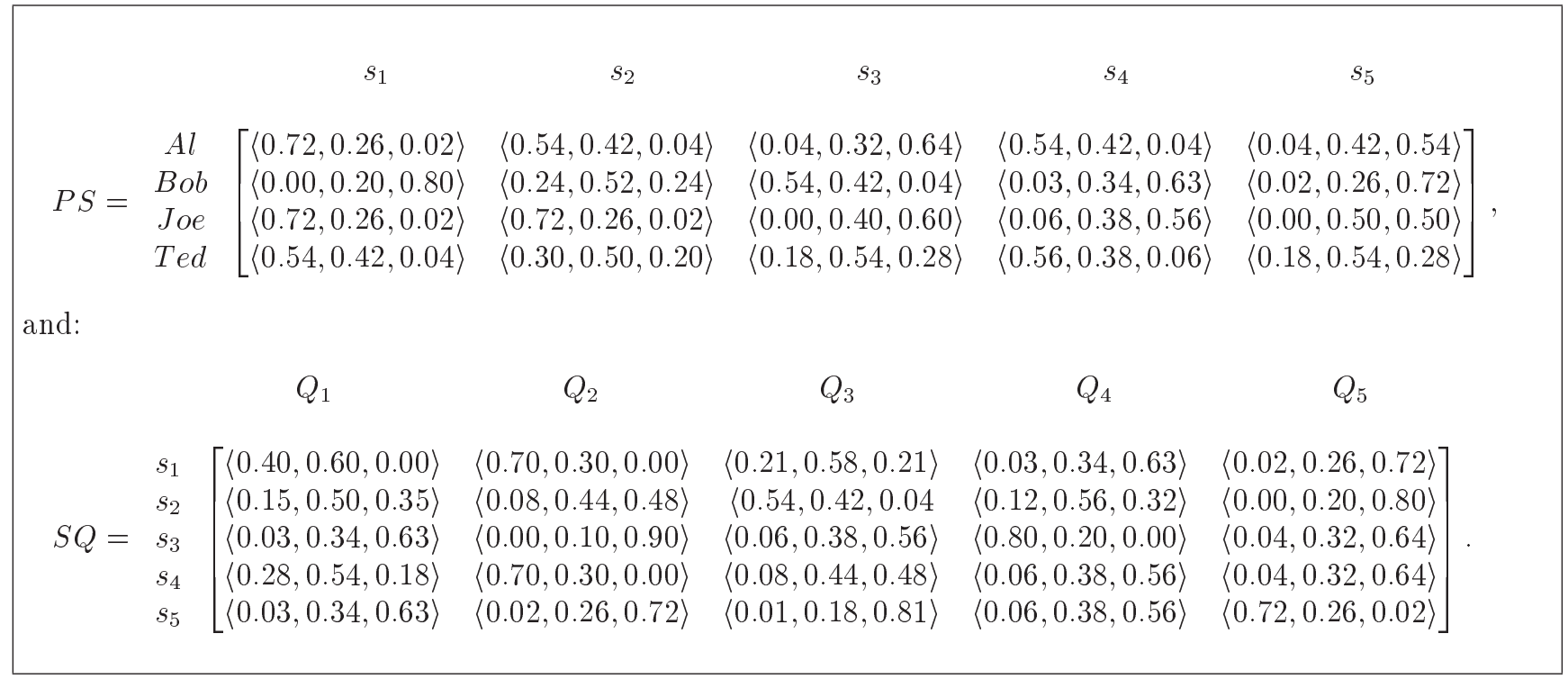

\section{Box XI}

\begin{tabular}{|c|c|c|c|c|c|}
\hline & $Q_{1}$ & $Q_{2}$ & $Q_{3}$ & $Q_{4}$ & $Q_{5}$ \\
\hline$A l$ & {$[0.8360$} & 0.8914 & 0.8122 & 0.4833 & $0.4499]$ \\
\hline Bob & 0.3474 & 0.2892 & 0.5525 & 0.7846 & 0.5449 \\
\hline Joe & 0.7583 & 0.6816 & 0.8113 & 0.5167 & 0.4661 \\
\hline Ted & 0.8608 & 0.8493 & 0.7447 & 0.6135 & 0.5647 \\
\hline
\end{tabular}

Box XII

ing indices have been computed corresponding to it:

\begin{tabular}{|c|c|c|c|c|c|}
\hline & $Q_{1}$ & $Q_{2}$ & $Q_{3}$ & $Q_{4}$ & $Q_{5}$ \\
\hline$A l$ & 0.8790 & 0.8899 & 0.8102 & 0.4788 & $0.3930]$ \\
\hline$B o b$ & 0.6165 & 0.4308 & 0.7599 & 0.9541 & 0.6226 \\
\hline Joe & 0.8061 & 0.7054 & 0.8696 & 0.5279 & 0.4697 \\
\hline Ted & 0.8695 & 0.7949 & 0.7362 & 0.6033 & 0.4966 \\
\hline
\end{tabular}

On the other hand, if weights are assigned to 0.15 , $0.25,0.20,0.15$, and 0.25 corresponding to $Q_{i}(i=$ $1,2, \ldots, 5)$, respectively, then, by applying correlation coefficient $K_{3}$ as given in Eq. (11), the following values are obtained:

\begin{tabular}{|c|c|c|c|c|c|}
\hline & $Q_{1}$ & $Q_{2}$ & $Q_{3}$ & $Q_{4}$ & $Q_{5}$ \\
\hline$A l$ & {$[0.8806$} & 0.8691 & 0.8455 & 0.5275 & \\
\hline & & 0.50 & 0.78 & 0.9 & \\
\hline & 0.80 & 0.7025 & 0.87 & 0.5411 & \\
\hline & 0.8654 & 0.7741 & 0.7425 & 0.6501 & 0.5182 \\
\hline
\end{tabular}

Based on this analysis, it has been concluded that Al suffers from Malaria, Bob from Stomach problems, Joe from Typhoid, and Ted from Viral Fever.
In order to compare the performance of the proposed approach with those of some existing approaches under the IFS environment, a comparison analysis is conducted based on the different approaches as given by the authors in $[31,51,56,57]$. The results corresponding to these approaches are summarized as below:

(a) If the correlation coefficient measures, as proposed by Zeng and $\mathrm{Li}$ [51] given in Eq. (13), are applied to the considered problem, then the indices values, shown in Box XII, are obtained for the patients with respect to the diagnoses. Therefore, based on this analysis, it has been concluded that $\mathbf{A l}$ suffers from Malaria, Bob from Stomach problems, Joe from Typhoid, and Ted from Viral Fever;

(b) If the correlation coefficient, as proposed by $\mathrm{Xu}$ et al. [31], given in Eq. (15), is applied to the considered problem, then the indices values of the patients with respect to the diagnoses are summarized as shown in Box XIII. Thus, based on this analysis, it has been concluded that Al suffers from Malaria, Bob from Stomach problems, Joe from Typhoid and Ted from Viral Fever;

(c) If the distance measure, as proposed by Ejegwa 


\begin{tabular}{|c|c|c|c|c|c|c|}
\hline & $Q_{1}$ & $Q_{2}$ & $Q_{3}$ & $Q_{4}$ & $Q_{5}$ & \\
\hline$A l$ & {$[0.7721$} & 0.8377 & 0.7941 & 0.4779 & 0.40487 & \\
\hline$B o b$ & 0.2857 & 0.2741 & 0.4810 & 0.6910 & 0.5394 & \\
\hline Joe & 0.6806 & 0.6591 & 0.7708 & 0.4965 & 0.4315 & . \\
\hline Ted & 0.8190 & 0.7013 & 0.6692 & 0.5451 & 0.4464 & \\
\hline
\end{tabular}

\section{Box XIII}

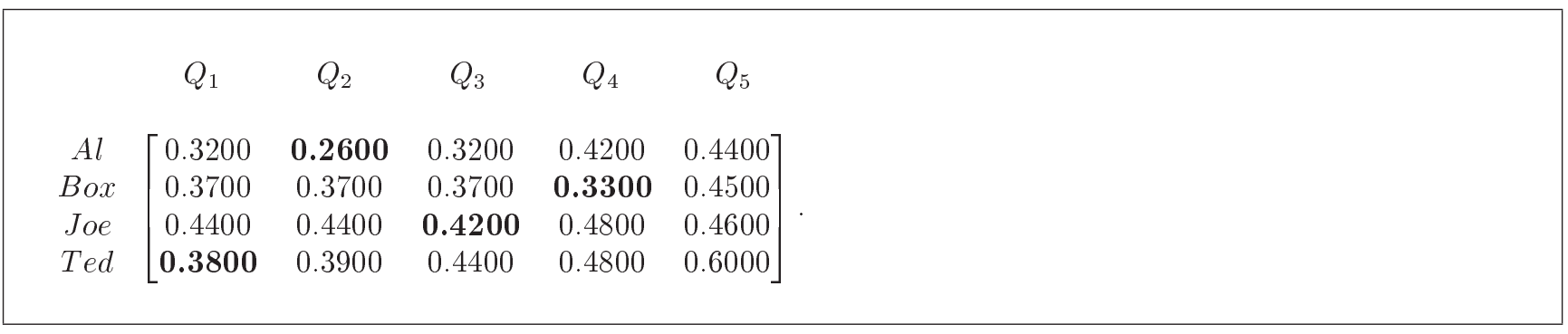

and Modom [56], is applied to the considered problem (for more details, see [56]), then measure values corresponding to each patient with respect to diagnoses $Q$ are obtained, summarized as shown in Box XIV. Therefore, based on this analysis, it has been concluded that Al suffers from Malaria, Bob from Stomach problems, Joe from Typhoid, and Ted from Viral Fever;

(d) If the similarity measure, as proposed by Hung and Yang [57], is applied to the considered problem, then their respective measure values are obtained as shown in Box XV. Thus, it has been observed that Al suffers from Malaria, Bob from Stomach problems, Joe from Typhoid, and Ted from Viral Fever.

\subsection{Advantages of the proposed method}

According to the above comparison analysis, the proposed method for addressing the decision-making problems has the following advantages:

(a) The IFS is characterized by the degrees of the membership and non-membership of an element such that their sum is less than 1. However, there may be a situation in which IFS theory be unable to provide the whole information about the situation. On the other hand, SPA theory provides an alternative way to deal with the certainty and uncertainty specific to quantitative analysis of "identity", "discrepancy", and "contrary" degrees of the connection number, such that the sum of their degrees is equal to one. Therefore, SPA theory is more suitable for real scientific and en gineering applications;

(b) It has been observed from the existing studies that various researchers $[31,51,52]$ proposed an algorithm using correlation coefficient for IFSs. As mentioned above, these correlation coefficients have some shortcomings, which cannot be represented by IFSs; hence, their corresponding analysis may not give its appropriate results;

(c) The proposed approach represents the intuitionistic fuzzy information using connection degrees, which can simultaneously describe the degrees of membership and non-membership as well as hesi-

\begin{tabular}{|c|c|c|c|c|c|}
\hline & $Q_{1}$ & $Q_{2}$ & $Q_{3}$ & $Q_{4}$ & $Q_{5}$ \\
\hline$A l$ & {$[0.8100$} & 0.8200 & 0.8000 & 0.5400 & 0.50007 \\
\hline$B o b$ & 0.6700 & 0.5400 & 0.7400 & 0.9000 & 0.6400 \\
\hline Joe & 0.7500 & 0.6800 & 0.8200 & 0.6000 & 0.5400 \\
\hline Ted & 0.8000 & 0.7700 & 0.7100 & 0.6300 & 0.5500 \\
\hline
\end{tabular}


tation degree with a simple mathematic depiction. Therefore, the connection degree can be computed without any transformation; hence, their corresponding proposed approach can effectively prevent any loss of information;

(d) The results obtained by the proposed methods might be more accurate since it considers the hesitation degree. The proposed correlation coefficient is more generalized and suitable to solve the reallife problem more accurately than the existing ones.

\section{Conclusion}

In the present manuscript, correlation coefficients under the set pair analysis theory were presented in terms of its parameters using the connection numbers. The shortcomings of the existing coefficients were highlighted and overcome by the proposed measures. The proposed correlation coefficients suitably utilized the degrees of certainty and uncertainties by the "identity", "discrepancy", and "contrary" degrees in terms of the connection number. Illustrative examples were provided which show that some of the existing correlation coefficients under intuitionistic fuzzy information fail to handle the situation; however, the proposed ones have easily identified it. Furthermore, to deal with the situations where the elements are correlated to each other, weighted correlation coefficients were defined. To demonstrate the efficiency of the proposed coefficients, examples from the field of pattern recognition as well as from the medical diagnosis were selected. Based on these studies, it was concluded that the proposed correlation coefficients could easily handle the reallife decision-making problems with their targets, hence being beneficial for the plant personal.

In the future, the technique can be extended to different fields and some new information measures are proposed. In addition, the methodology could be used to make further improvements to various mathematical programming models such as fuzzy goal programming, cluster analysis, dynamic programming and uncertain programming.

\section{References}

1. Zadeh, L.A. "Fuzzy sets", Inform. Control, 8, pp. 338353 (1965).

2. Atanassov, K.T. "Intuitionistic fuzzy sets", Fuzzy Sets Syst., 20, pp. 87-96 (1986).

3. Atanassov, K. and Gargov, G. "Interval-valued intuitionistic fuzzy sets", Fuzzy Sets Syst., 31, pp. 343-349 (1989).

4. Xu, Z.S. and Yager, R.R. "Some geometric aggregation operators based on intuitionistic fuzzy sets", Int. J. of Gen. Syst., 35, pp. 417-433 (2006).
5. Xu, Z.S. "Intuitionistic fuzzy aggregation operators", IEEE T. Fuzzy Syst., 15, pp. 1179-1187 (2007).

6. Garg, H. "Generalized intuitionistic fuzzy interactive geometric interaction operators using Einstein t-norm and t-conorm and their application to decision making”, Comput. Ind. Eng., 101, pp. 53-69 (2016).

7. Xu, Z. and Chen, J. "Approach to group decision making based on interval valued intuitionistic judgment matrices", Syst. Eng. - Theory and Pract., 27(4), pp. 126-133 (2007).

8. Xu, Z.S. "Methods for aggregating interval-valued intuitionistic fuzzy information and their application to decision making", Control and Decision, 22(2), pp. 215-219 (2007).

9. Garg, H., Agarwal, N., and Tripathi, A. "Entropy based multi-criteria decision making method under fuzzy environment and unknown attribute weights", Global J. Technol. Optimiz., 6, pp. 13-20 (2015).

10. Garg, H. "A new generalized improved score function of interval-valued intuitionistic fuzzy sets and applications in expert systems", Appl. Soft Comput., 38, pp. 988-999 (2016).

11. Garg, H. "Generalized Pythagorean fuzzy geometric aggregation operators using Einstein t-norm and tconorm for multicriteria decision-making process", Int. J. Intell. Syst., 32(6), pp. 597-630 (2017).

12. Garg, H. "A new generalized Pythagorean fuzzy information aggregation using Einstein operations and its application to decision making", Int. J. Intell. Syst., 31(9), pp. 886-920 (2016).

13. Garg, H. "Generalized intuitionistic fuzzy multiplicative interactive geometric operators and their application to multiple criteria decision making", Int. J. Mach. Learn. Cybern., 7(6), pp. 1075-1092 (2016).

14. Garg, H. "Some series of intuitionistic fuzzy interactive averaging aggregation operators", SpringerPlus, 5(1), p. 999 (2016). DOI: $10.1186 / \mathrm{s} 40064-016-2591-9$

15. Garg, H. "Confidence levels based Pythagorean fuzzy aggregation operators and its application to decisionmaking process", Computat. Math. Organization Theory, pp. 1-26, 23(4), pp. 546-571 (2017).

16. Wei, G.W. and Merigo, J.M. "Methods for strategic decision-making problems with immediate probabilities in intuitionistic fuzzy setting", Scientia Iranica, 19(6), pp. 1936-1946 (2012).

17. Nancy and Garg, H. "Novel single-valued neutrosophic decision making operators under frank norm operations and its application", Int. J. Uncertainty Quantif., 6(4), pp. 361-375 (2016).

18. Nancy and Garg, H. "An improved score function for ranking neutrosophic sets and its application to decision-making process", Int. J. Uncertainty Quantif., 6(5), pp. 377-385 (2016).

19. Yu, S.M., Wang, J., and Wang, J.Q. "An extended TODIM approach with intuitionistic linguistic numbers", Int. Trans. in Operational Research, 25(3), pp. 781-805 (2018). 
20. Garg, H., Agarwal, N., and Tripathi, A. "Some improved interactive aggregation operators under interval-valued intuitionistic fuzzy environment and its application to decision making process", Scientia Iranica: Transaction on Industrial Engineering, 24(5), pp. 2581-2604 (2017).

21. Wei, G. "Some induced geometric aggregation operators with intuitionistic fuzzy information and their application to group decision making", Appl. Soft Comput., 10, pp. 423-431 (2010).

22. Wang, J.Q., Han, Z.Q., and Zhang, H.Y. "Multi criteria group decision-making method based on intuitionistic interval fuzzy information", Group Decision and Negotiation, 23, pp. 715-733 (2014).

23. Wang, J.Q., Yang, Y., and Li, L. "Multi-criteria decision-making method based on single-valued neutrosophic linguistic Maclaurin symmetric mean operators", Neural Comput. Appl., pp. 1-19 (2016). DOI: http://dx.doi.org/10.1007/s00521-016-2747-0

24. Chiang, D.A. and Lin, N.P. "Correlation of fuzzy sets", Fuzzy Sets Syst., 102, pp. 221-226 (1999).

25. Liu, S.T. and Kao, C. "Fuzzy measures for correlation coefficient of fuzzy numbers", Fuzzy Sets Syst., 128, pp. 267-275 (2002).

26. Hong, D.H. "Fuzzy measures for a correlation coefficient of fuzzy numbers under $t_{w}$ (the weakest t-norm)based fuzzy arithmetic operations", Inform. Sci., 176, pp. 150-160 (2006).

27. Wang, G.J. and Li, X.P. "Correlation and information energy of interval-valued fuzzy numbers", Fuzzy Sets Syst., 103, pp. 169-175 (1999).

28. Gerstenkorn, T. and Manko, J. "Correlation of intuitionistic fuzzy sets", Fuzzy Sets Syst., 44, pp. 39-43 (1991).

29. Bustince, H. and Burillo, P. "Correlation of intervalvalued intuitionistic fuzzy sets", Fuzzy Sets Syst., 74, pp. 237-244 (1995).

30. Hong, D.H. "A note on correlation of interval-valued intuitionistic fuzzy sets", Fuzzy Sets Syst., 95, pp. 113117 (1998).

31. Xu, Z.S., Chen, J., and Wu, J.J. "Cluster algorithm for intuitionistic fuzzy sets", Inform. Sci., 178, pp. 3775-3790 (2008).

32. Xu, Z.S. "On correlation measures of intuitionistic fuzzy sets", Lect. Notes Comput. Sci., 4224, pp. 16-24 (2006).

33. Garg, H. "A novel correlation coefficients between Pythagorean fuzzy sets and its applications to decisionmaking processes", Int. J. Intell. Syst., 31(12), pp. 1234-1252 (2016).

34. Garg, H. "A novel accuracy function under intervalvalued Pythagorean fuzzy environment for solving multicriteria decision making problem", J. Intell. Fuzzy Syst., 31 (1), pp. 529-540 (2016).
35. Zhao, K. "Set pair and set pair analysis - a new concept and systematic analysis method", Proceedings of the National Conference on System Theory and Regional Planning, pp. 87-91 (1989).

36. Liu, C. , Zhang, L., and Yang, A. "The fundamental operation on connection number and its application", J. of Theoretical \& Applied Information Technology, 49(2), pp. 618-623 (2013).

37. Wang, J.Q. and Gong, L. "Interval probability stochastic multi-criteria decision-making approach based on set pair analysis", Control and Decision, 24, pp. 18771880 (2009).

38. Hu, J. and Yang, L. "Dynamic stochastic multi-criteria decision making method based on cumulative prospect theory and set pair analysis", Systems Engineering Procedia, 1, pp. 432-439 (2011).

39. Xie, Z., Zhang, F., Cheng, J., and Li, L. "Fuzzy multi-attribute decision making methods based on improved set pair analysis", 6th Int. Symposium on Computational Intelligence and Design, 2, pp. 386-389 (2013).

40. Fu, S. and Zhou, H. "Triangular fuzzy number multiattribute decision-making method based on set-pair analysis", J. of Software Engineering, pp. 1-7 (2016). DOI: $10.3923 /$ jse. 2016

41. Kumar, K. and Garg, H. "TOPSIS method based on the connection number of set pair analysis under interval-valued intuitionistic fuzzy set environment", Comput. Appl. Math., 37(2), pp. 1319-1320 (2018).

42. ChangJian, W. "Application of the set pair analysis theory in multiple attribute decision-making", J. of Mechanical Strength, 029, pp. 1009-1012 (2007).

43. Pan, Wu, K.Y., Jin, J.L., and Liu, X.W. "Assessment model of set pair analysis for flood loss based on triangular fuzzy intervals under $\alpha$-cut", Chinese Control and Decision Conference, pp. 3562-3567 (2009).

44. Zhang, Y., Yang, X.H., Zhang, L., Ma, W.Y., and Qiao, L.X. "Set pair analysis based on phase space reconstruction model and its application in forecasting extreme temperature", Math. Probl. Eng., 2013, Article ID 516150, 7 pages (2013).

45. Zhang, Y., Wang, S.G., and Xu, Y.T. "Online credit evaluation system based on analytic hierarchy process and set pair analysis", Int. Symposium on Computational Intelligence and Design, 1, pp. 453-456 (2008).

46. Yang, X.H., Di, C.L., He, J., Zhang, J., and Li, Y.Q. "Integrated assessment of water resources vulnerability under climate change in Haihe river basin", Int. J. Numer. Method Heat and Fluid Flow, 25(8), pp. 18341844 (2015).

47. Rui, Y., Zhongbin, W., and Anhua, P. "Multi-attribute group decision making based on set pair analysis", Int. J. of Advancements in Computing Technology, 4(10), pp. 205-213 (2012).

48. Sun, J., Li, L., Li, Y., and Liu, B. "Set pair analysis of lattice order decision-making model and application", J. of Chemical and Pharmaceutical Research, 6(4), pp. 52-58 (2014). 
49. Cao, Y.X., Zhou, H., and Wang, J.Q. "An approach to interval-valued intuitionistic stochastic multi-criteria decision-making using set pair analysis", Int. J. Mach. Learn. Cybern., 9(4), pp. 629-640 (2018).

50. Yang, J., Zhou, J., Liu, L., Li, Y., and Wu, Z., Similarity Measures Between Connection Numbers of Set Pair Analysis, Springer Berlin Heidelberg, Berlin, Heidelberg, pp. 63-68 (2008).

51. Zeng, W. and Li, H. "Correlation coefficient of intuitionistic fuzzy sets", J. Indust. Eng. International, 3(5), pp. 33-40 (2007).

52. Szmidt, E. and Kacprzyk, J. "Correlation of intuitionistic fuzzy sets", Lect. Notes Comput. Sci., 6178, pp. 169-177 (2010).

53. Ye, J. "Cosine similarity measures for intuitionistic fuzzy sets and their applications", Math. Comput. Model., 53, pp. 91-97 (2011).

54. Dengfeng, L. and Chuntian, C. "New similarity measure of intuitionistic fuzzy sets and application to pattern recognitions", Pattern Recogn. Lett., 23 , pp. 221-225 (2002).

55. Liu, H.W. "New similarity measures between intuitionistic fuzzy sets and between elements", Math. Comput. Model., 42, pp. 61-70 (2005).

56. Ejegwa, P.A. and Modom, E.S. "Diagnosis of viral hepatitis using new distance measure of intuitionistic fuzzy sets", Int. J. of Fuzzy Mathematical Archive, 8(1), pp. 1-7 (2015).

57. Hung, W.L. and Yang, M.S. "On similarity measures between intuitionistic fuzzy sets", Int. J. Intell. Syst., 23(3), pp. 364-383 (2008).

58. Son, L.H. and Thong, N.T. "Intuitionistic fuzzy recommender systems: An effective tool for medical diagnosis", Knowl.-Based Syst., 74, pp. 133-150 (2015).

\section{Biographies}

Harish Garg is an Assistant Professor at Thapar Institute of Engineering \& Technology (Deemed University), Patiala, India. Prior to joining this University,
Dr. Garg received a PhD in Applied Mathematics with specialization of reliability theory and soft computing techniques, from Indian Institute of Technology Roorkee, India in 2013 and an MSc in Mathematics from Punjabi University, Patiala, India in 2008. His research interests are in the field of computational intelligence, multi-criteria decision making problems, reliability theory, optimization techniques, various nature-inspired algorithms (e.g., genetic algorithms, swarm optimization), fuzzy and intuitionistic fuzzy set theories, and expert systems. Application areas include wide ranges of industrial and structural engineering design problems. Garg has authored/co-authored over 145 technical papers published in refereed International Journals. He has published seven book chapters. He also serves as an Editorial Board member of various International Journals. $\mathrm{He}$ is listed in International Who's Who of Professionals, Marquis Who's Who in the World, and Marquis Who's Who in Science and Engineering. In the year 2016, 2017 and 2018, Dr. Garg was privileged an awarded outstanding reviewer for the journals Applied Soft Computing, Applied Mathematical Modeling and Engineering Applications of Artificial Intelligence, Elsevier. He is the Associate Editor of Journal of Intelligent \& Fuzzy Systems. His Google citations are over $2350+$. For more details, visit http://sites.google.com/site/harishg58iitr/

Kamal Kumar is a $\mathrm{PhD}$ candidate at Thapar Institute of Engineering \& Technology (Deemed University), Patiala. He has obtained his master degree in Mathematics in 2014 and a Gold Medalist during this program. He has more than two years of teaching experience in different subjects of Engineering Mathematics. His current research interests are aggregation operation, multi-criteria decision-making, uncertainty theory, and intuitionistic fuzzy set theory. He has published 8 articles in the different reputed SCI journals. 